\title{
R\&D for the upgrade of the CMS muon system
}

\section{Marcello Abbrescia*i}

Universitá and Sezione INFN - via Amendola, 173 - 70126 Bari - ITALY

E-mail: marcello.abbrescialba.infn.it

The CMS muon system is based on three types of gaseous detectors, RPC, CSC and DT. While operating very well in the present conditions, upgrades are foreseen for each of the subsystems, necessary to guarantee its delicate role of muon triggering and tracking also in the High Luminosity phase of LHC, foreseen to start after Long Shutdown 3 in 2024 and to last for about 10 years.

Studies devoted to asses the system performance stability for the future will be presented, and the plans about the new DT and CSC electronics will be outlined. In addition, the strategy - which is being developed - to complement the existing system with new detectors, based on GEM or improved RPC technologies, will be shown.

The European Physical Society Conference on High Energy Physics

22-29 July 2015

Vienna, Austria

* Speaker.

${ }^{\dagger}$ on behalf of the CMS collaboration 


\section{Introduction}

The muon detector system of CMS consists of three types of muon detectors: Drift Tubes (DT), Cathode Strip Chambers (CSC), and Resistive Plate Chambers (RPC), as shown in Figure 1 and described in [1]. It was designed to operate in the initial LHC environment with a luminosity up to $10^{34} \mathrm{~cm}^{2} \mathrm{~s}^{-1}$, and provided excellent triggering, muon identification, timing, and momentum measurement during the entire Run 1 (see, for instance, [2] and [3]). Most of the system was installed in 2007, while the chambers in the fourth endcap disk (ME4/2, RE4/2 and RE4/3) were installed in 2014.

For the High Luminosity phase of LHC (HL-LHC in the following), foreseen to start in 2023 and to last for about ten years, many factors are to be taken into account, like the higher instantaneous and integrated luminosity, the possible detector degradations during the long periods of data taking, changes to the trigger structure and so on, all leading to the conclusion that it would be difficult to maintain the high level of performance achieved during Run 1 in the environment of HL-LHC without an extended program of upgrades for many of the system components. Details of the plans of the CMS collaboration can be found in the relative Technical Proposal [4].

Basically, there are three types of muon upgrades proposed for HL-LHC:

1. upgrades of the existing muon detectors and associated electronics, in order to ensure their longevity and good performance;

2. installation of additional muon detectors in the forward region $1.6<|\eta|<2.4$, already covered by the muon system, to increase redundancy and enhance the trigger and reconstruction capabilities;

3. extension of the muon system coverage for $|\eta|>2.4$, just behind the foreseen new endcap calorimeter, using additional muon detectors, to take advantage of the pixel tracking coverage extension.

Here an overview of these upgrades is presented, with particular emphasis on the R\&D currently undergoing to develop new detectors suitable for the harsh conditions foreseen during HLLHC.

\section{Upgrade of the existing detectors}

All detectors (DT, CSC and RPC) have started extensive campaigns of irradiation, in order to assess their longevity and performance stability up to an integrated luminosity of $3000 \mathrm{fb}^{-1}$. These are carried out at a new facility, the $\mathrm{GIF}^{++}$at CERN [5], which has come into operation at the beginning of 2015. At $\mathrm{GIF}^{++}$detectors and material samples can are irradiated by $662 \mathrm{keV}$ photons emitted by a $16.7 \mathrm{TBq}{ }^{137} \mathrm{Cs}$ source. A high-momentum particle muon beam extracted from the SPS is used to study the detectors performance on ionizing particles while these are irradiated with photons, simulating the high background conditions which will be present at CMS during HL-LHC. A lot of data have been accumulated in the past months, and preliminary results of projected performance stability are expected soon. 


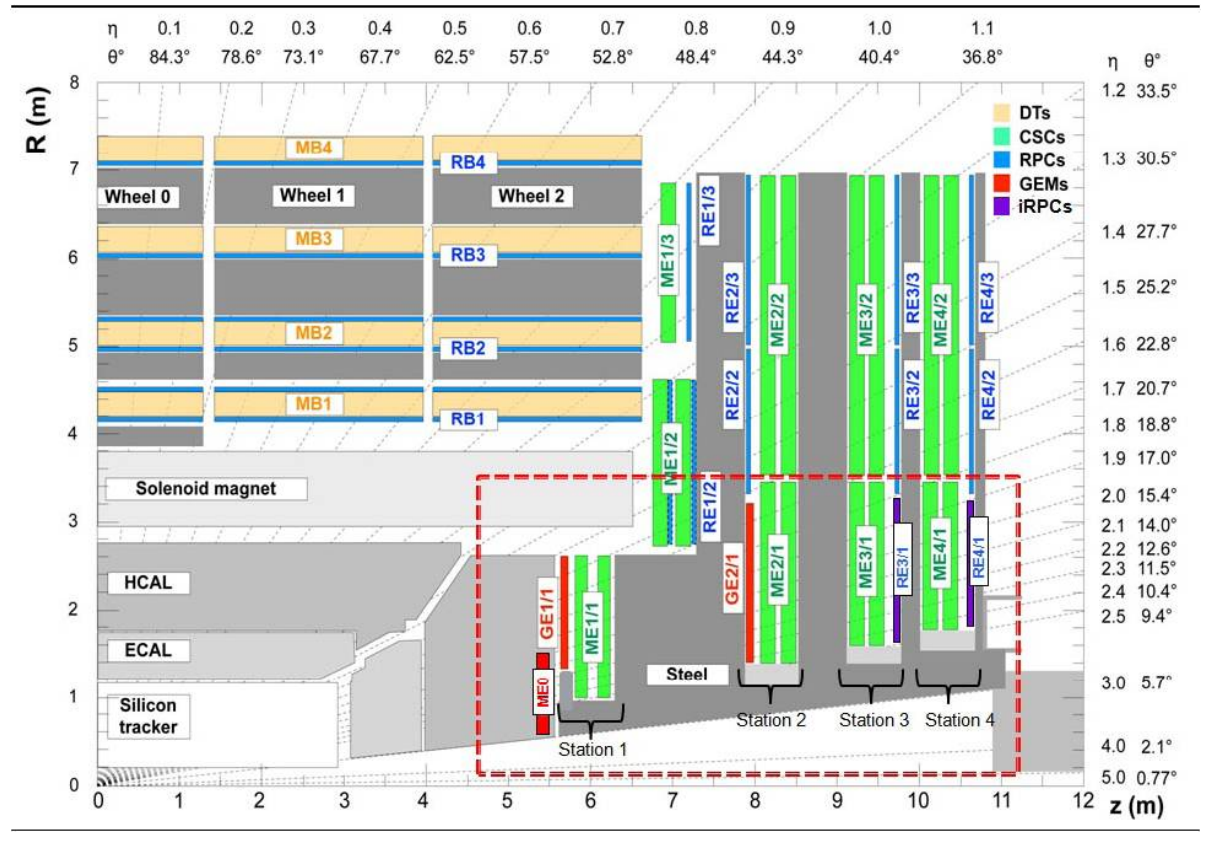

Figure 1: A quadrant of the muon system, showing DT chambers (yellow), RPC (light blue), and CSC (green). The locations of new forward muon detectors for HL-LHC are contained within the dashed box and indicated in red for GEM stations (ME0, GE1/1, and GE2/1) and dark blue for improved RPC stations (RE3/1 and RE4/1).

In addition to the above common mentioned aging studies, each sub-detector has foreseen specific upgrades.

DTs upgrade plans are mainly focused on electronics. It is known that DT electronics will need replacement due to limited radiation tolerance of some components; this replacement also gives the opportunity to increase the trigger rate capability and performance, and improve maintainability.

Each DT chamber houses the L1 Trigger and Readout electronics in an aluminum profile, called a Minicrate [6], which hosts the time digitization logic and the logic for the L1 trigger primitive generation. During HL-LHC the survival of this system is not guaranteed, and will not be able to cope with increasing the Level 1 trigger acceptance rate beyond $300 \mathrm{kHz}$, as it is foreseen. Consequently, the substitution of this electronics is planned for LS3, moving the trigger logic to the service cavern. Moreover, this replacement will allow the implementation of full time (and therefore space) resolution and to provide complete chamber information in the DT trigger system, with improved performance in terms of rate reduction and better matching with the tracker at the Level 1 trigger.

CSC upgrade plans are also focused on electronics, in particular foreseeing the replacement of cathode front-end boards (CFEB) on inner chambers in order to handle the increased L1 trigger latency and rate. The CFEB use analog charge storage within custom Switched Capacitor Array (SCA) chips (ASICs) that sample at 50 ns intervals and contain a depth of 96 cells. These boards are used on all CSCs except ME1/1 chambers which, in 2013-2014, have undergone an upgrade of the front- and back-end electronics to improve rate capability and performance at higher luminosity. With the increased L1 trigger latency (up to $12.5 \mathrm{~ms}$ ) and rate (up to $750 \mathrm{kHz}$ ) that is planned for 
HL-LHC CMS operation, the 108 inner CSC chambers (ME2/1, ME3/1, and ME4/1) will often fill the SCA cells; consequently no additional data can be stored, causing readout inefficiency.

Therefore, the CFEBs will be replaced by boards similar to the "digital" DCFEBs that have already been installed on the ME1/1 chambers. These boards flash-digitize the data continuously and store them in large digital buffers, resulting in zero dead-time and the capability to handle latencies well beyond the current specification. They also send their output data on $3.2 \mathrm{~Gb} / \mathrm{s}$ optical links, as compared to $1 \mathrm{~Gb} / \mathrm{s}$ achievable on the copper output cables of the CFEBs; the higher output data capability of the DCFEBs is important to handle the HL-LHC and CMS L1 trigger conditions.

Some additional elements of the CSC data acquisition system, namely the Data Acquisition Motherboards (DMBs) and Detector-Dependant Units (DDUs) that connect to the DCFEBs will will be replaced as well in order to handle the high data throughput using the fast optical links.

Among other activities, RPC will mainly focus on the investigation and qualification of a new gas mixture needed to fulfill new environmental regulations. The CMS RPC gas mixture is a three-component mixture of $95.2 \% \mathrm{C}_{2} \mathrm{H}_{2} \mathrm{~F}_{4}$ (Freon R-134a, tetrafluoroethane), $4.5 \% \mathrm{C}_{4} \mathrm{H}_{10}$ (isobutane) and $0.3 \% \mathrm{SF}_{6}$ (sulfur hexafluoride), humidified at about $40 \%$. Recently, because of its high Global Warming Potential (GWP) of 1430 (with respect to $\mathrm{CO}_{2}$ ), the use of R-134a is increasingly restricted by international regulations following the 1997 Kyoto Protocol.

Possible alternatives are already under study such as the R-152a, with a GWP of 120, and the most promising ones HFO-1234ze and HFO-1234yf (tetrafluoropropane) which are non-ozone depleting and with a very low GWP (6 and 4, respectively). R\&D tests with new gas mixtures have been recently started in some laboratories of the CMS collaboration, aimed at exploring these, and other, eco-gas candidates. Since the gas mixture is the core of a gaseous detector, replacing it is a non-trivial issue, and needs careful study to optimize again the detector working conditions with the new gas mixture. Moreover, in high-radiation environments like those expected at the HL-LHC, several new chemically-reactive impurities could be created inside the RPC, for instance $\mathrm{HF}$, which are potentially harmful to the detector materials and ultimately may degrade the detector performance. Therefore accurate measurements of production rate and design of the appropriate filters to remove them will be put in place.

\section{Additional muon detectors in the forward region}

Four stations, which are currently uninstrumented in the forward region of the muon system, are candidates to install there additional detectors to keep the overall performance of the system unchanged in the worse environmental conditions characteristic of HL-LHC. They are the closest to the beam pipe, where the background due, for instance, to neutrons and photons, is particularly intense, and are conventionally labeled GE1/1, GE2/1, and RE3/1, RE4/1 (see Figure 1).

Gas Electron Multipliers (GEM) detectors have been proposed for GE1/1 and GE2/1. The GEM detector is a micro-pattern gas amplification detector based on kapton foils coated on both sides with two conductive layers, and perforated with bi-conical holes which provide the amplification regions.

The GE1/1 and GE2/1 detectors are will be made each of a double layer of trapezoidal tripleGEM chambers. They will cover slightly more than 10 degrees in GE1/1 and 20 degrees in GE2/1, 
overlapping just like the corresponding CSC chambers in stations ME1/1 and ME2/1. For both endcaps, 72 superchambers will be needed for GE1/1 and 36 for GE2/1 (see Figure 2 and [7]).
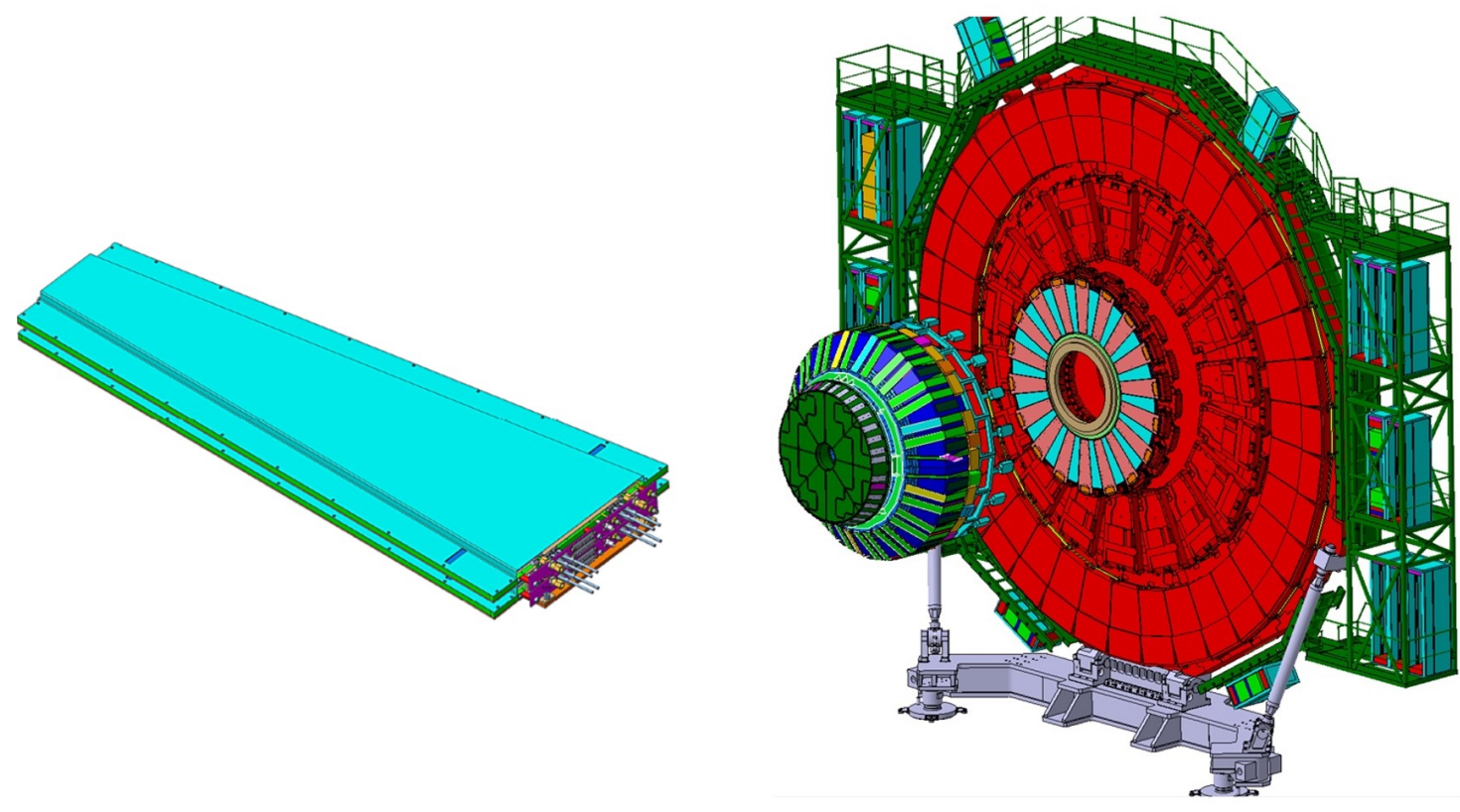

Figure 2: Left: A pair of GEM chambers form a superchamber. Right: First CMS muon endcap station where the inner ring is equipped with 18 long and 18 short triple GEM superchambers; long and short chambers are combined to maximize the instrumentation within given mechanical constraints in the endcap.

Several prototypes have been assembled and tested in the laboratory with x-rays and at test beams over the last five years. A complete description of the layouts of the various prototype and of the results obtained along the past years is reported in [8], where also a detailed description of the electronics, of the expected improved system performance, etc., is reported. In particular, it has been demonstrated that triple-GEM detector built with the single-mask technique, consisting in etching the kapton foils only from one side, can reach excellent performance, fully adequate for the proposed stations. In particular, a spatial resolution around $270 \mu \mathrm{m}$, compatible with the strip pitch, and a time resolution better than $5 \mathrm{~ns}$ can be reached [9]. Here, just as example, the efficiency for a triple GEM detector prototype, operated with an environmental friendly gas mixture is reported in Figure 3.

Early installation of the GE1/1 station in LS2 will address most of the causes of the trigger performance degradation. In addition to implementing the redundancy necessary for efficient selection of muon stubs in the most critical region, it will allow much improved measurement of the muon bending angle in the first station, using the lever arm formed by the GEMs and the CSCs (see Figure 4. This will greatly reduce the tails in the transverse momentum resolution, and therefore the trigger rate. Furthermore, the overall trigger efficiency will be improved with the additional hits provided by the new station. A plateau efficiency as high as $96 \%$ is expected even with a very preliminary version of the GEM-CSC integrated reconstruction algorithm. With this upgrade, CMS will be able to maintain a single-muon trigger threshold of pT $15 \mathrm{GeV}$, ensuring full efficiency for offline reconstruction of muons with $\mathrm{pT}>20 \mathrm{GeV}$. 


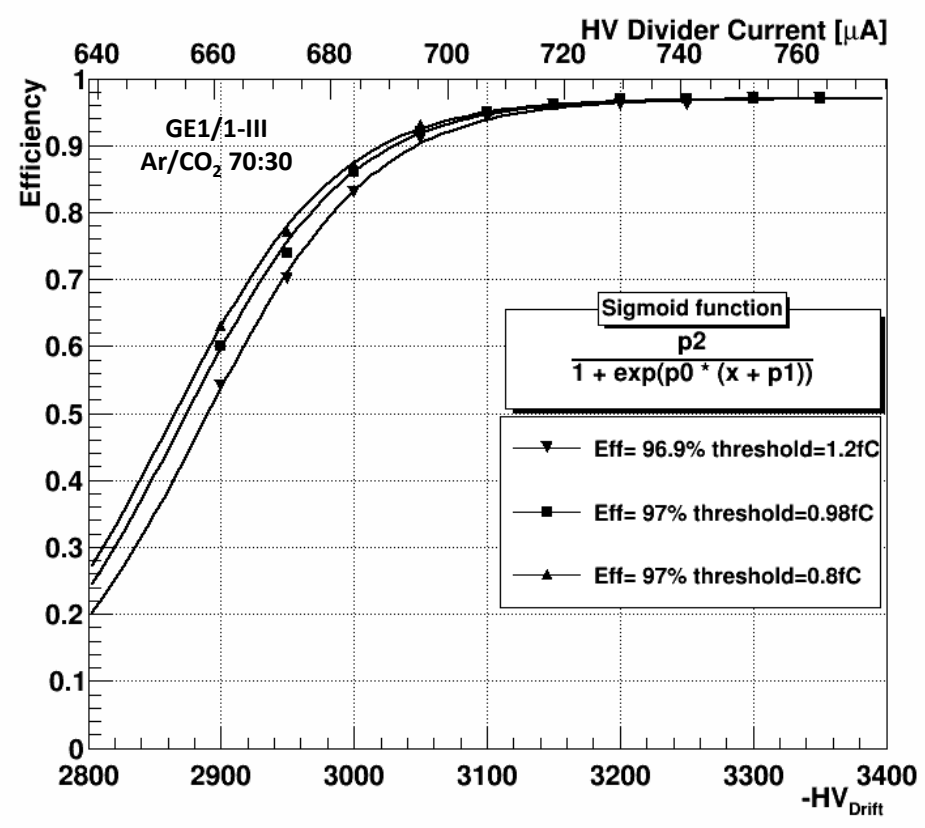

Figure 3: Eff. vs. HV applied to the drift electrode measured in central sector 5 of a GE1/1-III operated with $\mathrm{Ar} / \mathrm{CO}_{2} 70: 30$ and read out with APV chips. Three different cuts are applied offline to the strip charges to simulate VFAT2 threshold behavior and the resulting efficiency curves are fitted to sigmoid functions.
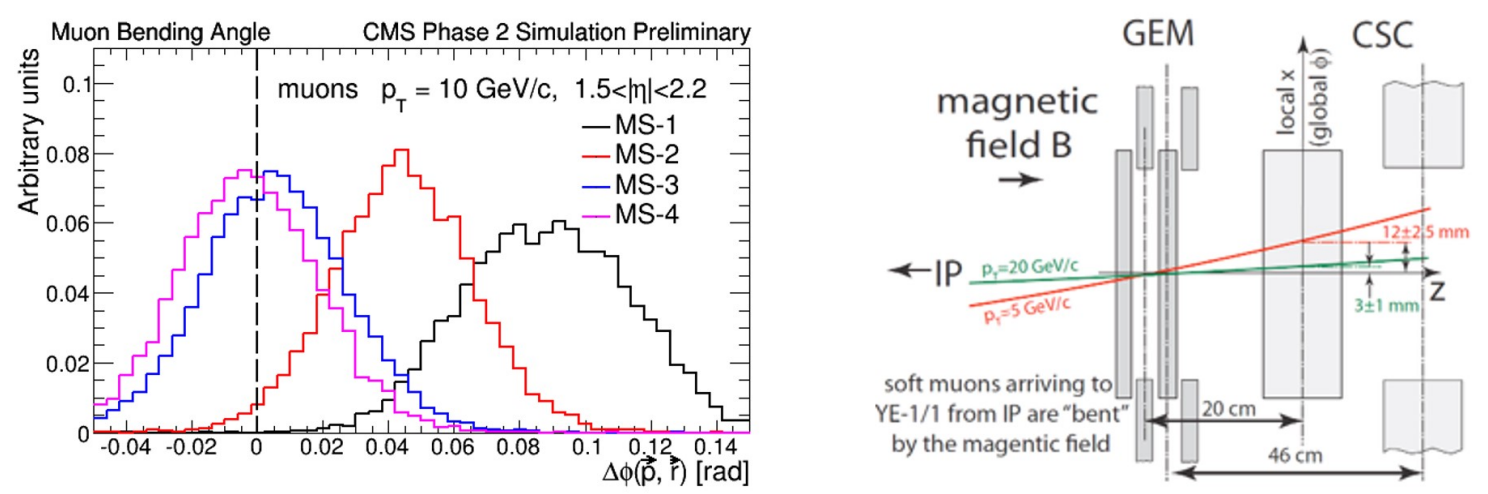

Figure 4: Left: Azimuthal bending angle of a simulated $10 \mathrm{GeV}$ muon with respect to a normal vector to a CSC chamber, comparing the distributions for the four stations. Right: Sketch of a measurement of the bending angle with a pair of a CSC and a GEM chamber, illustrating discrimination between lower and higher momentum muons.

For the RE3/1 and RE4/1 stations, an improved version of the present Resistive Plate Chambers, referred to as iRPC, capable of handling the particle rates foreseen during the HL-LHC phase, has been proposed (see Figure 5). The proposed RE3/1 and RE4/1 stations, schematically shown in Figure 1 , will cover the region $1.8<|\eta|<2.4$. Each of the 36 chambers, foreseen for both endcaps, will span 20 degrees each.

In particular, iRPC should be able to guarantee a rate capability around $2 \mathrm{kHz} / \mathrm{cm}^{2}$ and stability after integrating a charge corresponding to the full HL-LHC period, i.e. around $1-1.5 \mathrm{C} / \mathrm{cm}^{2}$. Many 
technological solutions are being considered: lower electrode resistivity, higher-amplification electronics, and improved detector configuration. The final technique adopted may combine the benefits of the different approaches.

One version of higher-amplification electronics has been developed in the framework of the muon system upgrade of the ATLAS experiment. Tests comparing the performance of CMS chambers equipped with the standard CMS electronics and an early prototype of this new electronics have been performed with cosmic rays; a shift of about $460 \mathrm{~V}$ in the efficiency curves is obtained (see [4]); the average charge corresponding to these voltages for $90 \%$ efficiency is reduced from $20 \mathrm{pC}$ to about $3 \mathrm{pC}$, a factor of 7 lower. This is a strong hint at an improved rate capability that is currently being be tested at the GIF++ facility.

The principle of a multi-gap HPL is shown in Figure 6 (left). The standard double-gap configuration used in CMS has been modified by adding an additional gap on both sides of the readout strips. The thickness of each of the four gaps is $1 \mathrm{~mm}$. In this particular case, the material used for the electrodes is identical to the one already used for CMS/RPC chambers (same resistivity), and the readout electronics as well. Efficiency for cosmic rays muon vs. operating voltage in the two cases of no irradiation and irradiation with a $150 \mathrm{mCi}{ }^{137} \mathrm{Cs} \gamma$-ray source providing a hit rate around $3 \mathrm{kHz} / \mathrm{cm}^{2}$ on the whole chamber is shown in Figure 6 (right). The chamber reaches full efficiency and has a plateau several hundreds volts wide in both cases where the probability of a streamer is acceptably low $(<10 \%)$, satisfying the requirement on rate capability. Several other results are described in detail in [12].

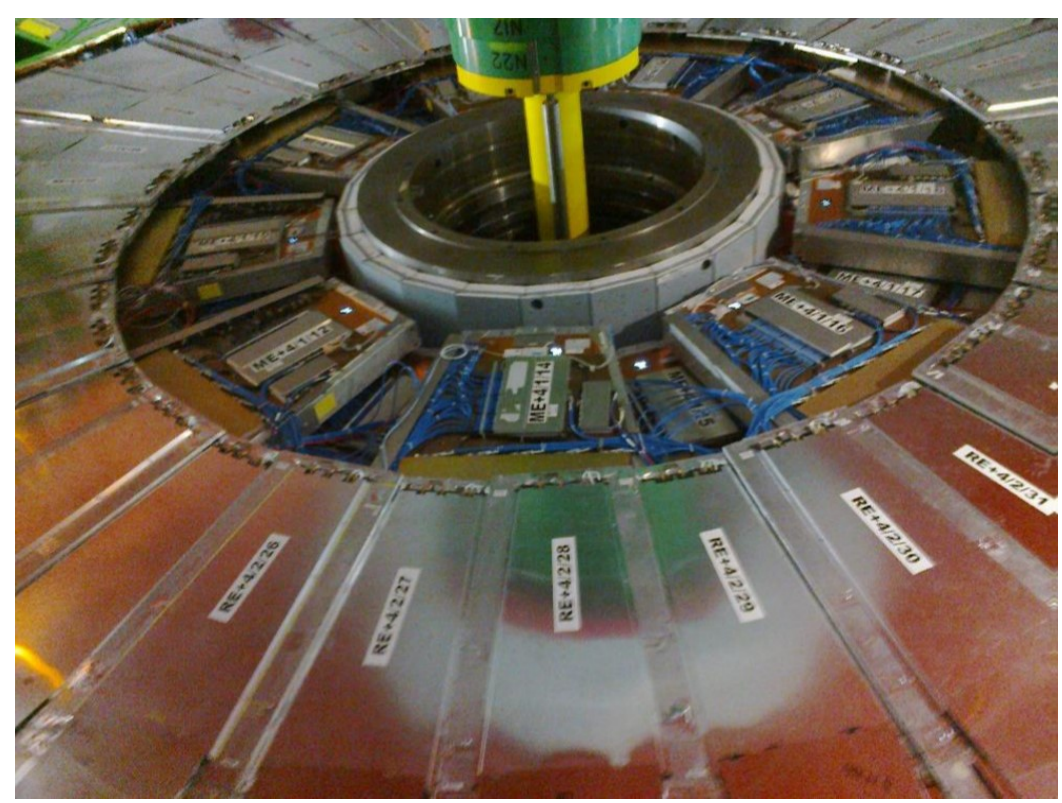

Figure 5: Picture of the inner part of the third endcap station of the Muon system of the CMS experiment, which is being proposed to be instrumented with improved RPCs.

Again, reasons that drive to install these new stations for the HL-LHC are described in details in [4]. A qualitative indication that additional RPC chambers are needed in the forward region to overcome performance issues at high luminosity is presented in Figure 7. It shows the average number of measuring muon hits associated with a global muon track, as a function of $\eta$ for the 

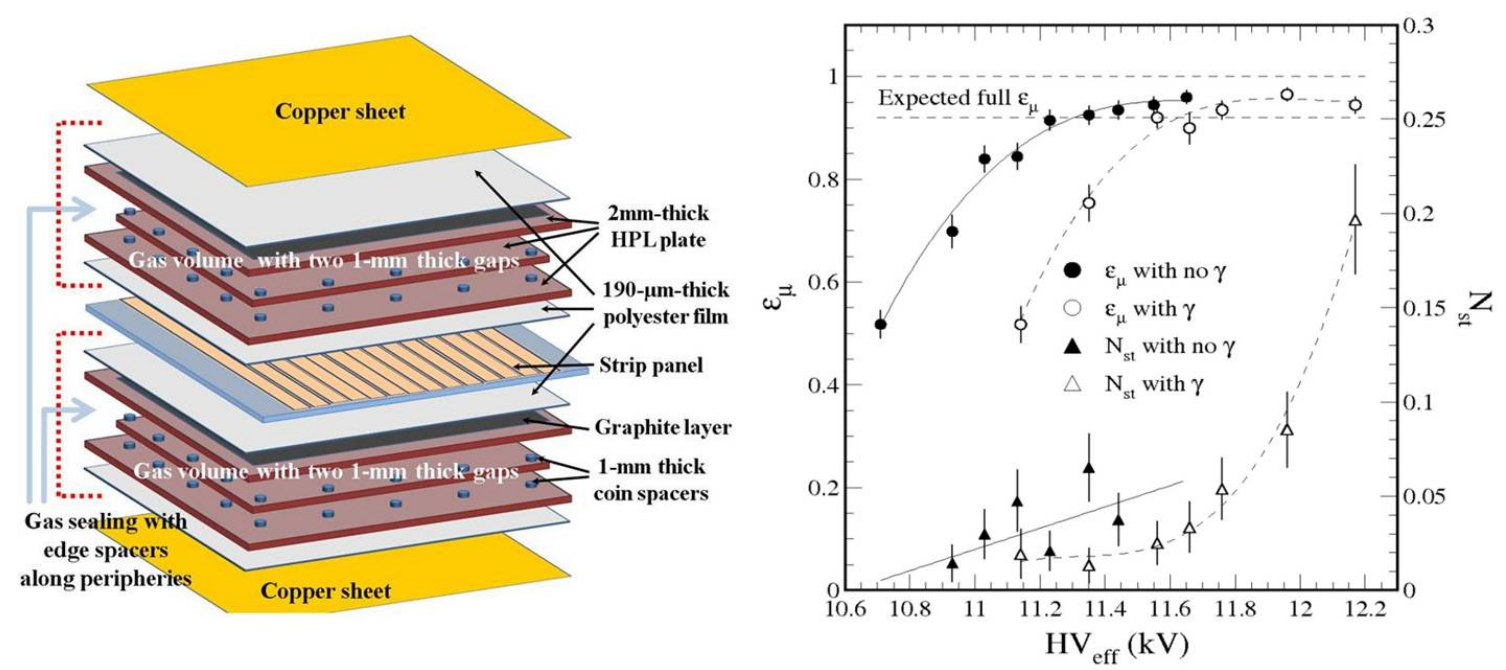

Figure 6: Left: view of a multigap HPL RPC prototype. Right: efficiency plateau (circles) and streamer probability (triangles) vs. operating voltage with (open symbols) and without (solid symbols) a $3 \mathrm{kHz} / \mathrm{cm} 2$ g irradiation.

Phase-I muon detector (black histogram). While in the barrel muon detector, with $\eta<0.8$, muon tracks are associated with an average of 25 hits, this number decreases to only 18 in the forward region with $\eta>1$.6. In the same figure, the colored curves illustrates the rate of one of the major backgrounds, the neutron flux through the first station of the muon system. It undergoes a drastic increase (note the logarithmic scale on the right) with increasing $|\eta|$, and other backgrounds such as the flux of low- $p_{T}$ muons also exhibit a similar trend. The muon detector redundancy is therefore currently the least in the region where the backgrounds are the largest. With two additional hits in each GEM detector and one per iRPC, the number of hits will become similar over the full coverage of the muon system.

\section{Extension to $|\eta|>2.4$}

The ME0 station will be installed in a space of $30 \mathrm{~cm}$ freed behind the new endcap calorimeter as shown in Figure 8. The coverage is foreseen to extend up to $|\eta|=3$, or more, depending on the boundary defined by the calorimeter. The configuration described here extends up to a value of 3 , taking into account the shielding that will be required at the inner radius. The lower $\eta$ boundary of ME0 is affected by substantial mechanical infrastructure such as bolts and plates that must be located near the periphery of the endcap nose. The detector is expected to supply good muon ID in offline analysis.

GEM detectors are able to handle the $10 \div 100 \mathrm{kHz} / \mathrm{cm}^{2}$ rates and the integrated doses of $5 \mathrm{C} / \mathrm{cm}^{2}$ expected in this region during HL-LHC. At the moment, it has been proposed a sixlayer device to allow proper rejection of neutron background (see Figure 8). The design of ME0 incorporates neutron and photon shielding using, for example, borated polyethylene and lead. The detector will be made of wedges with inner and outer radii of 300 and $1495 \mathrm{~mm}$. 


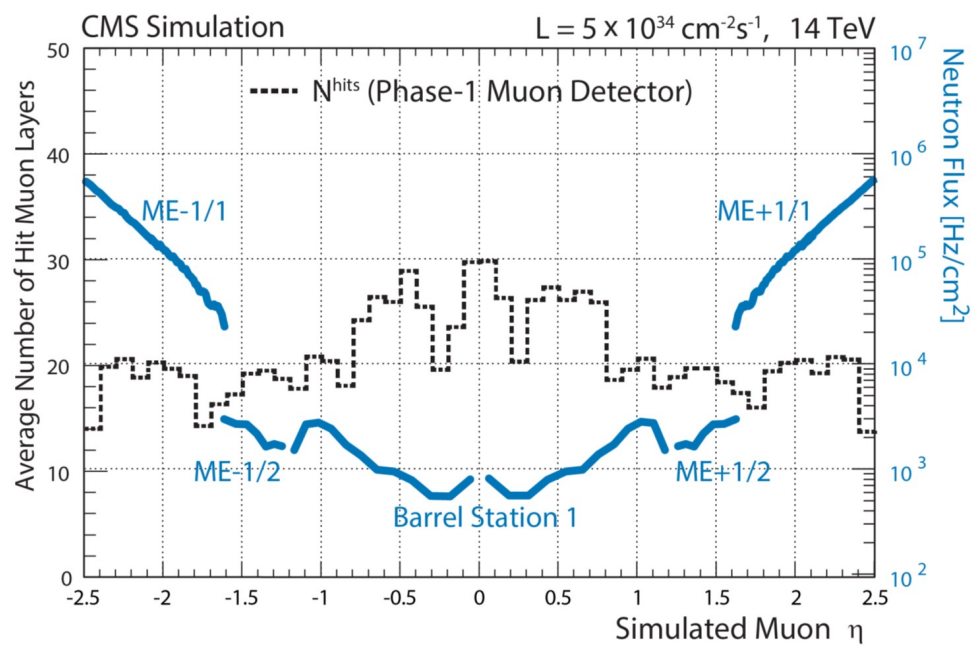

Figure 7: The dashed line shows the average number of muon layers with reconstructed hits for a simulated muon as a function of $\mathrm{h}$. It is compared to the flux of neutrons in $\mathrm{Hz} / \mathrm{cm} 2$ shown as colored curves (note the log scale on the right), which are the dominant cause of background hits, for the muon station first crossed by a muon with a given $\mathrm{h}$. Forward region is exposed to the highest rates in the system, yet has the fewest muon layers needed for offline and trigger reconstruction and momentum measurement. Depending on the detector type the conversion factor can vary somewhat, but typically the hit rate is of the order of 0.2 neutron flux.

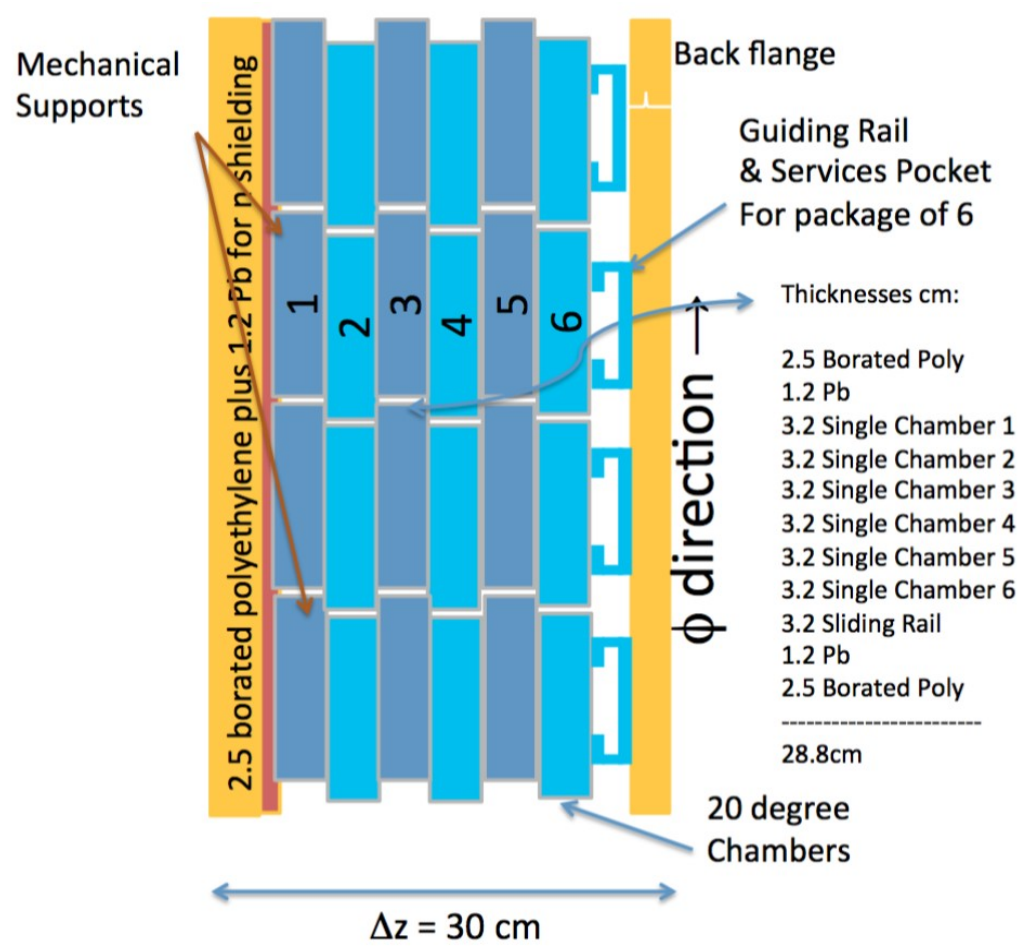

Figure 8: A preliminary layout of approximate 100 of a 6-layer ME0 layout in f-z view, using 2024 tripleGEM chambers in the $30 \mathrm{~cm}$ space made available behind the new Endcap Calorimeters. 


\section{Conclusions}

During Run 1, the CMS Muon systems has been operating extremely well, delivering good data for physics. At the wake of Run 2 the detector performance is within specifications both for triggering and as a reconstruction system.

Nevertheless, keeping the system performance at the same level also during HL-LHC will be a considerable challenge. Efforts to consolidate the present system in order to assure its longevity also during HL-LHC have already started.

In addition, new detector technologies are proposed to be installed in still empty locations. Triple-GEMs are proposed to instrument the $1.5<|\eta|<2.4$ region of the CMS forward muon system with high spatial resolution detectors that complement the existing Cathode Strip Chambers detectors, providing redundancy, enhanced triggering and reconstruction capabilities; an additional station, called ME-0 is proposed behind the calorimeters, to increase pseudorapidity coverage.

In RE3/1 and RE4/1, an improved version of RPCs capable to handle the high particle rates foreseen during the HL-LHC phase will complement the existing ME3/1 and ME4/1 stations, currently instrumented with CSC only, providing robustness to the L1 muon trigger where redundancy is missing, and ensuring constant performance even during HL-LHC.

\section{References}

[1] CMS collaboration, CMS The Muon Project Technical Design Report, CERN-LHCC-1008-032, CMS-TDR-3

[2] CMS Collaboration, Performance of CMS muon reconstruction in pp collision events at sqrt( $(s)=7$ TeV, JINST 7 (2012) P10002.

[3] CMS collaboration, The performance of the CMS muon detector in proton-proton collisions at $\operatorname{sqrt}(s)=7 \mathrm{TeV}$ at the LHC, JINST 7 (2013) P100002.

[4] CMS Collaboration, Technical Proposal for the Phase-II upgrade of the Compact Muon Solenoid, CERN-LHCC-2015-10, LHCC-P-008, CMS-TDR-15-02

[5] M. Capeans et al., Proposal to the CERN SPSC: A GIF++ Gamma Irradiation Facility at the SPS H4 Beam Line, SPSC-2009-029 / SPSC-P-339 (2009).

[6] CMS collaboration, CMS Technical Design Report for the Trigger and Data Acquisition project, Volume I, CERN-LHCC-2000-038, CMS-TDR-6.1 (2000) page.

[7] CMS collaboration, CMS Technical Design Report for the Muon Endcap GEM upgrade, CERN-LHCC-2015-012, CMS-TDR-013

[8] D. Abbaneo et al., Characterization of GEM Detectors for Application in the CMS Muon Detection System, Nucl. Sci. Symp. Conf. Rec. 1416-1422 (2010).

[9] D. Abbaneo et al., Test Beam Results of the GE1/1 Prototype for CMS High- $\eta$ Muon System Future Upgrade, IEEE Nucl.Sci.Symp.Conf.Rec. 1806-1810 (2011).

[10] M. Abbrescia et al., Study of long-term performance of CMS RPC under irradiation at the CERN GIF, Nucl. Instrum. Meth. A533 (2004) 102.

[11] H.C. Kim et al., Quantitative aging study with intense irradiation tests for the CMS forward RPCs, Nucl. Instrum. Meth. A602 (2009) 771. 
[12] K. S Lee at al., Rate capability study for a four-gap phenolic RPC with a CS-137 source, in print on JINST, contribution to RPC2014. 